Pacific Journal of 


\title{
ON UNIVERSAL EXTENSIONS OF DIFFERENTIAL FIELDS
}

\author{
E. R. KOLCHIN
}

Dedicated to Gerhard Hochschild on the occasion of his 65th birthday

The main result of this paper is the following:

Theorem: Let $\mathscr{U}$ be a universal extension of the differential field $\mathscr{T}$ of characteristic zero and let $\mathscr{G}$ be a strongly normal extension of $\mathscr{F}$ in $\mathscr{C}$. Then $\mathscr{C}$ is a universal extension of $\mathscr{G}$.

Introduction. We deal with differential fields, always of characteristic zero, relative to a nonempty finite set of commuting derivation operators. By an extension of a differential field, we always mean a differential field extension. An extension $\mathscr{F}^{-1}$ of a differential field $\mathscr{F}$ is said to be finitely generated if $\mathscr{F}^{-1}$ has a finite subset $\Phi$ such that $\mathscr{F}^{\prime}=\mathscr{F}\langle\Phi\rangle=$ the smallest extension of $\mathscr{F}$ in $\mathscr{F}^{\prime}$ that contains $\Phi$.

Let $\mathscr{F}$ be a differential field. Recall that an extension $\mathscr{Q} 6$ of

is called universal if, for any finitely generated extension $\mathscr{F}_{1}$ of $\mathscr{F}$ in $\mathscr{C}$ and any finitely generated extension $\mathscr{P}$ of $\mathscr{F}_{1}$ not necessarily in $\mathscr{Q}, \mathscr{F}$ can be embedded in $\mathscr{Q}$ over $\mathscr{F}_{1}$, i.e., there exists an extension of $\mathscr{F}_{1}$ in $\mathscr{C}$ that is isomorphic (in the sense of differential fields) to $\mathscr{C}$ over $\mathscr{F}_{1}$. Such a universal extension of $\mathscr{F}$ always exists ([2] p. 132, Th. 2). It is not unique, but if $\mathscr{C}$ and $\mathscr{T}$ are two universal extensions of $\mathscr{F}$, then there exist universal extensions $\mathscr{C}^{\prime}$ and $\mathscr{C}^{\prime}$ of $\mathscr{F}$, lying in $\mathscr{C}$ and $\mathscr{F}$, respectively, such that $\mathscr{C}^{\prime}$ is isomorphic to $\mathscr{C}^{\prime}$ over $\mathscr{F}$ ([2] p. 135, Exerc. 7).

Let $\mathscr{C}$ be a universal extension of the differential field $\mathscr{F}$ and let $\mathscr{O}$ be an extension of $\mathscr{F}$ in $\mathscr{Z}$. Under favorable conditions, $\mathscr{Q}$ is then a universal extension of $\mathscr{G}$, too. For example, this is the case when $\mathscr{S}$ is finitely generated over $\mathscr{F}$ ([2] p. 133, Prop. 4), and also when $\mathscr{G}$ is algebraic over $\mathscr{F}$ ([2] p. 134, Exerc. 1). The main purpose of the present note is to point out another such favorable condition. We shall show $(\S 1)$ that when $\mathscr{C}$ is a strongly normal extension of $\mathscr{F}$, in the general sense of Kovacic [4] (i.e., not necessarily finitely generated), then $\mathscr{C}$ is universal over $\mathscr{C}$. This result shows that, in the study of strongly normal extensions, it is not necessary to replace 26 by a larger universal extension of $\mathscr{F}$ (see Kovacic [4] p. 518).

Every strongly normal extension of $\mathscr{F}$ in $\mathscr{C}$ is embeddable over $\mathscr{F}$ in a constrained closure of $\mathscr{F}$ in $\mathscr{C}$ ([3] p. 162, Th. 3 or Blum [1] p. $42(15)$ ) and hence, in particular, is constrained over $\mathscr{F}$ 
([3] p. 148, Th. 1). It is tempting to conjecture that the above result generalizes to constrained extensions of $\mathscr{F}$ in $\mathscr{C}$. We shall show (\$2) by a counterexample that $\mathscr{C}$ can fail to be universal over a constrained closure of $\mathscr{F}$ in $\mathscr{K}$.

1. Strongly normal extensions. Recall ([2] p. 393), for a finitely generated extension $\mathscr{C}$ of $\mathscr{F}$ in a given universal extension $\mathscr{U}$ of $\mathscr{F}$, that $\mathscr{G}$ is called strongly normal over $\mathscr{F}$ if every isomorphism $\sigma$ over $\mathscr{F}$ of $\mathscr{G}$ onto an extension of $\mathscr{F}$ in $\mathscr{C}$ is strong, i.e., has the property that $\sigma c=c$ for every constant $c$ in $\mathscr{G}$ and $\mathscr{G} \mathscr{K}=\sigma \mathscr{G} \cdot \mathscr{K}$, where $\mathscr{K}$ denotes the field of constants of $\mathscr{Z}$. This definition is apparently a relative one, depending on the universal extension $\mathscr{U}$ of $\mathscr{F}$ in which $\mathscr{G}$ is embedded. It is eary to see, however, that if $\mathscr{G}$ is strongly normal over $\mathscr{F}$ relative to one $\mathscr{C}$, then $\mathscr{G}$ is strongly normal over $\mathscr{F}$ relative to every $\mathscr{C}$, so that the notion of strongly normal finitely generated extension is an absolute one. When $\mathscr{G}$ is not necessarily finitely generated over $\mathscr{F}, \mathscr{G}$ is said, following Kovacic [4] p. 518, to be strongly normal over $\mathscr{F}$ if $\mathscr{G}$ is the union of strongly normal finitely generated extensions. Hence, also this more general notion is absolute.

It follows from [2] pp. 402-403, Th. 5, and the definition that if $\mathscr{G}$ is any strongly normal extension of $\mathscr{F}$ and $\mathscr{E}$ is any extension of $\mathscr{F}$, both contained in an extension of $\mathscr{F}$ having the same field of constants as $\mathscr{F}$, then $\mathscr{C} \mathscr{E}$ is a strongly normal extension of $\mathscr{E}$, and $\mathscr{G}$ and $\mathscr{E}$ are linearly disjoint over $\mathscr{G} \cap \mathscr{E}$.

We now prove the main theorem of this paper which was stated in the opening paragraph.

Proof. (a) We must show that if $\mathscr{G}_{1}$ is a finitely generated extension of $\mathscr{G}$ in $\mathscr{C}$ and $\mathscr{H}$ is any finitely generated extension of $\mathscr{G}_{1}$ not necessarily in $\mathscr{U}$, then there exists an embedding $\mathscr{H} \rightarrow \mathscr{C}$ over $\mathscr{G}_{1}$. As before, denote the field of constants of $\mathscr{C}$ by $\mathscr{K}$, and put $\mathscr{C}=\mathscr{F} \cap \mathscr{K}, \mathscr{C}_{1}=\mathscr{G}_{1} \cap \mathscr{K}$. Then $\mathscr{C}=\mathscr{G} \cap \mathscr{K}^{([2]}$ p. 393, Prop. 9), $\mathscr{C}_{1}$ is a finitely generated field extension of $\mathscr{C}$ ([2] p. 113, Cor. 1 to Prop. 14), $\mathscr{\mathscr { C }}$ is a universal extension of $\mathscr{F} \mathscr{C}_{1}$, and $\mathscr{G} \mathscr{C}_{1}$ is a strongly normal extension of $\mathscr{F} \mathscr{C}_{1}$ ([2] p. 396, Th. 2). Thus, we may replace $\left(\mathscr{F}, \mathscr{G}, \mathscr{G}_{1}, \mathscr{H}\right)$ by $\left(\mathscr{F} \mathscr{C}_{1}, \mathscr{G}_{1}, \mathscr{G}_{1}, \mathscr{H}\right)$, i.e., we may suppose that $\mathscr{F}, \mathscr{G}, \mathscr{G}_{1}$ have the same field of constants $\mathscr{C}$.

(b) That being the case, fix a finite family $\beta$ of generators of $\mathscr{G}_{1}$ over $\mathscr{G}$. Then $\mathscr{C}$ is a universal extension of $\mathscr{F}\langle\beta\rangle$ and $\mathscr{G}_{1}=$ $\mathscr{G} \mathscr{F}\langle\beta\rangle$ is a strongly normal extension of $\mathscr{F}\langle\beta\rangle$. Thus, we may replace $\left(\mathscr{F}, \mathscr{G}, \mathscr{G}_{1}, \mathscr{C}\right)$ by $\left(\mathscr{T}\langle\beta\rangle, \mathscr{G}_{1}, \mathscr{G}_{1}, \mathscr{C}\right)$, i.e., we may suppose that $\mathscr{G}_{1}=\mathscr{G}$. 
(c) That being the case, let $\mathscr{D}$ denote the field of constants of if. Then $\mathscr{D}$ is a finitely generated field extension of $\mathscr{C}$, so that there exists an isomorphism $\mathscr{D} \approx \mathscr{D}^{\prime}$ over $\mathscr{C}$ with $\mathscr{D}$ ' a field extension of $\mathscr{C}$ in $\%$. Because $\mathscr{C}$ and $\mathscr{O}$ are linearly disjoint over fo ([2] p. 87, Cor. 1 to Th. 1), and likewise $\mathscr{G}$ and $\mathscr{D}^{\prime}$, this can be extended to an isomorphism $\mathscr{G} \mathscr{D} \approx \mathscr{C} \mathscr{D}^{\prime}$ over $\mathscr{G}$. This can in turn be extended to an isomorphism $\mathscr{K}^{\prime} \approx \mathscr{K}^{\prime}$, where $\mathscr{K}^{\prime}$ is a finitely generated extension of $\mathscr{C} \mathscr{D}^{\prime}$ not necessarily in $\mathscr{Z}$. Now, $\mathscr{C}$ is a universal extension of $\mathscr{F}^{\prime}, \mathscr{C} \mathscr{D}^{\prime}$ is a strongly normal extension of $\mathscr{F} \mathscr{D} '$ in $\mathscr{C}$, and $\mathscr{C} '$ is a finitely generated extension of $\mathscr{G} \mathscr{D} '$ with field of constants $\mathscr{D}^{\prime}$. An embedding $\mathscr{K}^{\prime} \rightarrow \mathscr{C}$ over $\mathscr{G} \mathscr{D}^{\prime}$ would, when composed with the isomorphism $\mathscr{C}^{\prime} \approx \mathscr{C}^{\prime}$ over $\mathscr{G}$, yield an embedding $\mathscr{C} \rightarrow \mathscr{C}$ over $\mathscr{S}$. Thus, we may replace $(\mathscr{G}, \mathscr{C})$ by $\left(\mathscr{O} \mathscr{O}^{\prime}, \mathscr{G} \mathscr{D}^{\prime}, \mathscr{K}^{\prime}\right)$, i.e., we may suppose that the field of constants of $\mathscr{C}$ is $\mathscr{C}$.

(d) That being the case, fix a finite family $\alpha$ of generators of the extension $\mathscr{K}$ of $\mathscr{B}$, and put $\mathscr{E}=\mathscr{F}\langle\alpha\rangle$. Then $\mathscr{C} \cap \mathscr{E}$ is a finitely generated extension of $\mathscr{F}$ ([2] p. 112, Prop. 14), so that $\mathscr{C}$ is universal over $\mathscr{F} \cap \mathscr{E}$. Thus, we may replace $(\mathscr{F}, \mathscr{C}, \mathscr{C}, \mathscr{E}$ ) by $(\mathscr{F} \cap \mathscr{C}, \mathscr{C}, \mathscr{C}, \mathscr{O})$, i.e., we may suppose that $\mathscr{G} \cap \mathscr{F}=\mathscr{F}$. Since $\because$ is strongly normal over $\mathscr{F}$, then the differential field $\mathscr{C}=\mathscr{G} \mathscr{E}$ is strongly normal over $\mathscr{E}$ and $\mathscr{O}$ and $\mathscr{E}$ are linearly disjoint over

(e) Because $\%$ is universal over there exists an isomorphism $\mathscr{F} \approx \mathscr{E}_{0}$ over with $\mathscr{E}_{0}$ an extension of $\mathscr{F}$ in $\not \mathscr{l}$, and this isomorphism can be extended to an isomorphism $\sigma: \mathscr{K} \approx K_{0}$, where $\because i$ is an extension of $\mathscr{F}$ (and of $\mathscr{E}_{0}$ ) not necessarily in $\mathscr{H}$. Put $\mathscr{S}_{0}=\sigma \mathscr{G}$. Then $\mathscr{H}_{0}^{\prime}=\mathscr{S}_{0} \mathscr{E}_{0}$, this differential field is a strongly normal extension of $\mathscr{E}_{0}$, and $\mathscr{G}_{0}$ and $\mathscr{E}_{0}$ are linearly disjoint over $\mathscr{F}$. Evidently $\mathscr{H}$ is universal over $\mathscr{B}_{0}$ (because $\mathscr{E}_{0}$ is finitely generated over $\mathscr{F}$ ), and hence the strongly normal extension $\mathscr{G}_{0} \mathscr{E}_{0}$ of $\mathscr{S}_{0}$ can be embedded in $\mathscr{H}$ over $\mathscr{E}_{0}$, i.e., there exists an isomorphism $\sigma_{0}: \mathscr{G}_{0} \mathscr{F}_{0} \approx \mathscr{G}_{2} \mathscr{C}_{0}$ over $\mathscr{E}_{0}$ with $\sigma_{0} \mathscr{G}_{0}=\mathscr{G}_{2} \subset \not / h$. The field of constants of $\mathscr{G}_{2} \mathscr{E}_{1}$, like those of $\mathscr{K}_{0}=\mathscr{G}_{0} \mathscr{E}_{0}$ and $\mathscr{C}=\mathscr{C} \mathscr{C}$, is $\mathscr{C}$, and hence $\mathscr{S}_{2} \mathscr{F}_{0}$ and $\mathscr{\mathscr { C }}$ are linearly disjoint cover 6 . Therefore $\mathscr{F}_{2} \mathscr{F}_{0}$ and $\mathscr{G}_{2} \mathscr{\mathscr { C }}$ are linearly disjoint over $\mathscr{F}_{2}$. But by $(\mathrm{d}), \mathscr{O}$ and $\mathscr{G}$ are linearly disjoint over $\mathscr{F}$, so that $\mathscr{E}_{0}$ and $\mathscr{G}_{0}$ are, too, and hence also $\mathscr{G}_{1}$ and $\mathscr{G}_{2}$. Therefore $\mathscr{E}_{0}$ and $\mathscr{G}_{2} \mathscr{\mathscr { C }}$ are linearly disjoint over $\mathscr{F}$. But $\mathscr{G}$ is strongly normal over $\mathscr{F}$, so that $\mathscr{G} \subset \sigma_{0} \sigma \mathscr{G} \cdot \mathscr{K}=\mathscr{Y}_{2} \mathscr{Y}$. Hence $\mathscr{E}_{0}$ and $\mathscr{S}$ are linearly disjoint over $\mathscr{F}$. Therefore, $i d_{\mathscr{E}_{0}}$ and the isomorphism $\mathscr{G} \approx \mathscr{G}$ (restriction of $\left.\left(\sigma_{0} \circ \sigma\right)^{-1}\right)$ extend to an isomorphism $\tau: \mathscr{S}_{2} \mathscr{F}_{11} \approx \mathscr{O}_{0}$. The composite isomorphism $\tau \circ \sigma_{0} \circ \sigma$ is an embedding of $\mathscr{Y}$ into $\mathscr{C}$ over $\mathscr{C}$. 
2. A counterexample for constrained extensions. Recall that an extension $\mathscr{G}$ of a differential field is said to be constrained ([3] p. 144) if every finite family of elements of $S$ is constrained over $\mathscr{F}$ in the sense of [2] p. 142, that a differential field is said to be constrainedly closed ([3] p. 145) if it has no constrained extension other than itself, and that $\mathscr{G}$ is said to be a constrained closure of $\mathscr{F}$ ([3] p. 147) if $\mathscr{C}$ is constrainedly closed and is embeddable over closed $\mathscr{F}$ in every constrainedly extension of $\mathscr{F}$. A constrained closure of $\mathscr{F}$ always exists, and it is a constrained extension of $\mathscr{F}$.

We are going to exhibit an ordinary differential field $\mathscr{F}$, a universal extension $\mathscr{C}$ of $\mathscr{F}$, and an extension $\mathscr{F}$ of $\mathscr{F}$ in $\mathscr{C}$ such that $\mathscr{F}$ is a constrained closure of $\mathscr{F}$ and $\mathscr{Z}$ is not universal over S.

Let $\%$ be any denumerable field of characteristic zero and put $\mathscr{F}=\mathscr{C}(x)=$ the field of rational fractions over $\%$ in an indeterminate $x$; $F$ has a unique structure of ordinary differential field with field of constants $C$ in which the derivative of $x$ is 1 . By [3] p. 149, Prop. 4, we may fix a denumerable universal extension $\mathscr{C}$ of By [3] p. 146, Cor. 1 to Prop. 3, Z6 is constrainedly closed.

The set of solutions in $\% \frac{}{6}$ different from 0 and 1 of the differential equation

$$
y^{\prime}=y^{\prime \prime}-y^{2}
$$

is denumerable and hence can be arranged in a sequence

$$
\eta_{0}, \eta_{1}, \eta_{12}, \cdots
$$

By [3] $\S 8$, this set is infinite and is an independent set of conjugates over $\mathscr{F}$, and $\mathscr{F}\left\langle\eta_{0}, \eta_{1}, \eta_{2}, \cdots\right\rangle$ is constrained over $\mathscr{F}$ (see [3] p. 144, Prop. 1). Because $Z_{6}$ is constrainedly closed, $\left\langle\eta_{0}, \eta_{1}, \eta_{2}, \cdots\right\rangle$ has a constrained closure $\mathscr{C}$ in $\%$. The differential ideal $\left[y^{\prime}-y^{3}+y^{2}\right]$ of the differential polynomial algebra $\mathscr{C}\{y\}$ is evidently prime and does not have a generic zero in $\mathscr{C}$ (because all its zeros in $\mathscr{C}$ are in $\mathscr{O}$ ). Therefore, $\mathscr{Z}$ is not universal over $\mathscr{C}$. (The same argument shows that $\mathscr{H}_{6}$ is even not universal over $\left\langle\eta_{0}, \eta_{1}, \eta_{2}, \cdots\right\rangle$.) We are going to show that $\mathscr{G}$ is a constrained closure of $\mathscr{F}$.

By [3] p. 144, Prop. 2(a), $\mathscr{G}$ is constrained over $\mathscr{F}$. Let be any denumerable constrained closure of (e.g., any constrained closure of $\mathscr{F}$ in $\mathscr{C}$ ). The set of solutions in $\mathscr{Y}$ of the above differential equation can be arranged in a sequence

$$
\check{\zeta}_{n}, \check{\zeta}_{1}, \check{\zeta}_{2}, \cdots \text {. }
$$

As before, this set is infinite and is an independent set of conjugates over. F. Therefore, there exists an isomorphism 


$$
\varphi: \mathscr{F}\left\langle\eta_{0}, \eta_{1}, \eta_{2}, \cdots\right\rangle \approx \mathscr{F}\left\langle\zeta_{0}, \zeta_{1}, \zeta_{2}, \cdots\right\rangle .
$$

Now, $\mathscr{F}\left\langle\zeta_{0}, \zeta_{1}, \zeta_{2}, \cdots\right\rangle$ is normal over $\mathscr{F}$ in $\mathscr{C}$ (see [3] $\S 6$ p. 153). Hence, by [3] p. 159, Cor. 1 to Th. 2, $\mathscr{H}$ is a constrained closure of $\mathscr{F}\left\langle\zeta_{0}, \zeta_{1}, \zeta_{2}, \cdots\right\rangle$. Therefore, by [3] p. 158, Th. 2(b), $\varnothing$ can be extended to an isomorphism $\mathscr{G} \approx \mathscr{H}$, so that $\mathscr{G}$ is a constrained closure of $\mathscr{F}$.

\section{REFERENCES}

1. Lenore Blum, Differentially closed fields: a model-theoretic tour, Contributions to Algebra, Academic Press, New York, 1977, pp. 37-61.

2. E. R. Kolchin, Differential Algebra and Algebraic Groups, Academic Press, New York, 1973.

3. 141-170.

4. Jerald Kovacic, Pro-algebraic groups and the Galois theory of differential fields, Amer. J. Math., 95 (1973), 507-536.

Received March 13, 1978. Research supported by a grant from the National Science Foundation.

Columbia University

New York, NY 10027 



\section{PACIFIC JOURNAL OF MATHEMATICS}

\section{EDITORS}

DONALD BABBITT (Managing Editor)

University of Galifornia

Los Angeles, California 90024

HUGo RossI

University of Utah

Salt Lake City, UT 84112

C. C. MOORE AND ANDREW OGG

University of California

Berkeley, CA 94720
J. DUGUNDJI

Department of Mathematics University of Southern California Los Angeles, California 90007

R. FINN AND J. MILGRAM Stanford University Stanford, California 94305

\section{ASSOCIATE EDITORS}

E. F. BECKENBACH

B. H. NEUMANN

F. WOLF

K. YosHIDA

\section{SUPPORTING INSTITUTIONS}

UNIVERSITY OF BRITISH COLUMBIA CALIFORNIA INSTITUTE OF TECHNOLOGY UNIVERSITY OF CALIFORNIA MONTANA STATE UNIVERSITY UNIVERSITY OF NEVADA, RENO NEW MEXICO STATE UNIVERSITY OREGON STATE UNIVERSITY UNIVERSITY OF OREGON
UNIVERSITY OF SOUTHERN CALIFONIA STANFORD UNIVERSITY UNIVERSITY OF HAWAII UNIVERSITY OF TOKYO UNIVERSITY OF UTAH WASHINGTON STATE UNIVERSITY UNIVERSITY OF WASHINGTON 


\section{Pacific Journal of Mathematics}

\section{Vol. 86, No. $1 \quad$ November, 1980}

Gert Einar Torsten Almkvist, Invariants, mostly old ones .............. 1

Hyman Bass, Groups of integral representation type ................ 15

A. Białynicki-Birula, On action of SL(2) on complete algebraic

varieties........................................

Frederick Paul Greenleaf and Martin Allen Moskowitz, Groups of

automorphisms of Lie groups: density properties, bounded orbits, and

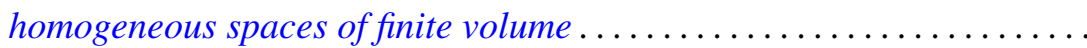

Raymond Taylor Hoobler, A cohomological interpretation of Brauer groups

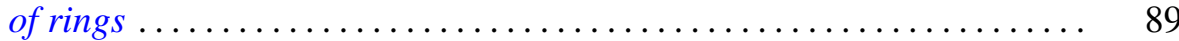

Irving Kaplansky, Superalgebras ........................ 93

Jerrold Lewis Kleinstein and Alex I. Rosenberg, Succinct and

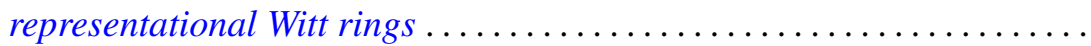

E. R. Kolchin, On universal extensions of differential fields ............ 139

Andy R. Magid, Analytic subgroups of affine algebraic groups. II ....... 145

Calvin Cooper Moore, The Mautner phenomenon for general unitary

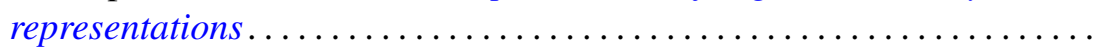

George Daniel Mostow, On a remarkable class of polyhedra in complex hyperbolic space ................................ 171

Brian Lee Peterson, Extensions of pro-affine algebraic groups. II . . . . . . 277

John Henry Reinoehl, Lie algebras and affine algebraic groups......... 287

Maxwell Alexander Rosenlicht, Differential valuations .

John Brendan Sullivan, The second Lie algebra cohomology group and Weyl modules..................................... 321

Moss Eisenberg Sweedler, Right derivations and right differential

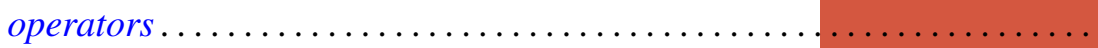

Bostwick Frampton Wyman, Time varying linear discrete-time systems. II. Duality.................................. 